\title{
Actitud, satisfacción y lealtad de los clientes en las Cajas Municipales del Perú
}

\section{Attitude, satisfaction and loyalty of customers in Municipal Savings Banks of Peru}

Dr. Edwin Hernán Ramírez-Asís es Doctor en Administración. Jefe de la unidad de Investigación de la Facultad de Administración y Turismo de la Universidad Nacional Santiago Antúnez de Mayolo, Perú (ehramireza@unasam.edu.pe) (https://orcid.org/0000-0002-9918-7607)

Dr. Misael Erikson Maguiña-Palma es docente e investigador de la Universidad Norbert Wiener (Perú) (mmaguia@hotmail.com) (https://orcid.org/0000-0002-4545-7413)

Mg. Rosario Mercedes Huerta-Soto es docente de la Universidad Nacional Santiago Antúnez de Mayolo, Huaraz (Perú) (mhuertas@unasam.edu.pe) (https://orcid.org/0000-0002-1738-3437)

\section{Resumen}

El propósito del estudio fue extender el modelo SERVQUAL adicionando la actitud del cliente a las tres principales dimensiones como componentes de la medición de la calidad del servicio e investigar la influencia en la satisfacción y la lealtad de los clientes en el sector microfinanciero, específicamente de las cajas municipales de ahorro y crédito que poseen agencias en la Región Ancash, Se utilizó la técnica de la encuesta donde se recopilaron datos de 391 clientes a través del cuestionario mediante el muestreo aleatorio simple. Por tratarse de una metodología de modelos de ecuaciones estructurales (SEM) para evaluar el modelo teórico se empleó la técnica de análisis de Mínimo Cuadrado Parcial (PLS) mediante el uso del software Smart PLS 3.3.0. El hallazgo reveló que el modelo extendido tiene un impacto significativo en la satisfacción y la lealtad de los clientes en las cajas municipales de ahorro y crédito. Los coeficientes de determinación fueron; satisfacción del cliente $(\mathrm{r} 2=0.637)$ y lealtad del cliente $(\mathrm{r} 2=0.510)$, con un error cuadrático medio de aproximación (SRMR) de 0.06 que hace relevante el modelo confirmatorio. Además, los resultados de este estudio serán útiles para que los gerentes y los encargados de formular políticas mejoren la calidad del servicio en las cajas municipales. Se recomienda extender este estudio en otros países en vías de desarrollo, ya que se contextualizó en la realidad del sistema financiero peruano.

\begin{abstract}
The purpose of the study was to extend the SERVQUAL model by adding the customer's attitude to its three main dimensions as one more component of measuring service quality and to investigate the causal relationship in customer satisfaction and loyalty in the microfinance sector, specifically in the municipal savings banks. and credit that have agencies in the Ancash Region, the survey technique was used, where data was collected from 391 clients through the questionnaire through simple random sampling. As it is a structural equation modeling (SEM) methodology, the partial least square (PLS) analysis technique was used to evaluate the theoretical model using the Smart PLS 3.3.0 software, the finding revealed that the extended model has a significant impact on customer satisfaction and loyalty in municipal savings banks and credit, the coefficients of determination were; customer satisfaction $(r 2=0.637)$, customer loyalty $(r 2=0.510)$ with a mean squared error of approximation (SRMR) of 0.06 , the results make the confirmatory model relevant. Furthermore, the results of this study will be useful for managers and policymakers to improve the quality of service in municipal savings banks. It is recommended to extend this study in other developing countries, since it was contextualized in the reality of the Peruvian financial system.
\end{abstract}

\section{Palabras clave I keywords}

Tangibilidad, fiabilidad, seguridad, actitud del cliente, satisfacción del cliente, microfinanzas, modelo SEM, Smart PLS.

Tangibility, reliability, security, customer attitude, customer satisfaction, microfinance, SEM model, Smart PLS.

Cómo citar: Ramírez-Asís, E.H., Maguiña-Palma, M.E., y Huerta-Soto, R.M. (2020). Actitud, satisfacción y lealtad de los clientes en las Cajas Municipales del Perú. Retos Revista de Ciencias de la Administración y Economía,10(20), pp. 329-343. https://doi.org/10.17163/ret.n20.2020.08 


\section{Introducción}

Las cajas municipales de ahorro y crédito (CMAC) forman parte del sistema financiero peruano desde 1982 y son un soporte muy importante en el desarrollo económico y social del país. Actualmente son 12 cajas municipales: CMAC Arequipa, Huancayo, Piura, Cusco, Trujillo, Sullana, Ica, Tacna, Maynas, Paita, Del Santa, y Lima. Además, cuentan con agencias a nivel nacional e incluso son reguladas por la superintendencia de banca y seguro y administradora de fondo de pensiones (SBS), también poseen el 40.2\% del mercado microfinanciero peruano (Escobedo et al., 2019). Así mismo, ofertan servicios financieros minorista de colocaciones y captaciones para empresarios y personas naturales que no pueden acceder al sistema bancario (Geraldo et al., 2020). Existen instituciones similares en Latinoamérica, como las Cooperativas de Ahorro y Crédito en Ecuador que es una clara muestra de la necesidad de instituciones microfinancieras que promuevan la inclusión financiera (Salinas, 2011).

La Región Ancash se localiza al oeste del Perú, limita por el norte con La Libertad, al sur con la Región Lima, al este con la Región Huánuco y por el oeste con el Océano Pacífico. Ubicada a una altitud media de 2688 metros sobre el nivel del mar; incluye las provincias de Caraz, Yungay, Carhuaz, Huaraz y Chimbote. Con una población de 1213900 habitantes es la octava región más poblada y la sexta economía del Perú por contribuir al Producto Bruto Interno - PBI:3,5 \%. Por esta razón, el mercado de la Región Ancash es muy atractivo para las cajas municipales de ahorro y crédito, donde operan hace más de 20 años y con buenos resultados, en la actualidad la principal preocupación de los gerentes de las CMAC's es la conservación de los clientes tratando de mejorar la calidad de sus servicios (Escobedo et al., 2019).

Los servicios en este grupo de instituciones financieras se están convirtiendo cada vez más en un factor competitivo y se consideran una herramienta indispensable para el flujo de ingresos de las microempresas (Escobedo et al., 2019). Se evidencian esfuerzos para mejorar la calidad de sus servicios y satisfacer las necesidades de sus clientes, pero la diversidad de sus clientes no lo hace una tarea fácil (Morillo et al., 2011). Por otro lado, los clientes determinan la calidad en función a las diferencias de las expectativas del servicio esperado y de la percibidas de lo que entrega el ofertante (Parasuraman et al., 1988). Además, Vargas y Aldana (2014) definen la calidad del servicio como el resultado de la comparación que el consumidor realiza entre sus expectativas y sus percepciones. En el sector financiero, la calidad del servicio juega un papel vital en la mejora de la satisfacción del cliente (Arancibia et al., 2013). Adicionalmente, Berdugo-Correa, et al. (2016) mencionan que otorgar un servicio de calidad generaría un buen nivel de satisfacción y además una relación a largo plazo con el cliente.

Con respecto al sector financiero Alnaser et al. (2018), describió que las instituciones financieras con ventajas competitivas mantienen una fuerte relación con sus clientes y esto proporciona niveles favorables de lealtad con los clientes. También, los gerentes perciben que la calidad del servicio puede aumentar el rendimiento de una empresa (Guerrero, 2014; Ureña et al., 2016). Sin embargo, la calidad del servicio y la satisfacción del cliente son términos intercambiables (González, 2015). Además, se ha demostrado que en el sector financiero existe una relación positiva de la calidad del servicio y la satisfacción de los clientes bancarios (Alnaser et al., 2018). En efecto, se han realizado varios estudios en el sector servicios para comprender las dimensiones de la calidad del servicio y la satisfacción del cliente (Morillo et al., 2011). 
El rápido crecimiento de los servicios financieros ha proporcionado diferentes alternativas a los clientes (León, 2018). Por ello, la calidad del servicio juega un papel importante para mejorar las ganancias, la cuota de mercado, el desarrollo de una buena imagen y proporcionar una ventaja competitiva (Rodríguez, 2014). En este sentido, no existe un consenso respecto a la calidad de servicio y es muy complejo su medición en el sector financiero, por consiguiente, el presente estudio pretende medir la calidad del servicio mediante cuatro variables latentes, demostrar su influencia en la satisfacción del cliente y su impacto en la lealtad de los clientes de las cajas municipales de ahorro y crédito del Perú.

Si bien la calidad de servicio ha sido abordada por muchos investigadores, existen muy pocos estudios donde se relaciona la calidad, la satisfacción y la lealtad de los clientes en el sector de servicios financieros. Adicionalmente hasta el momento no se ha incluido la actitud del cliente como parte del modelo SERVQUAL, por consiguiente, se propone considerar la actitud del cliente como parte del modelo SERVQUAL, y así complementar la medición de la calidad de servicio en los clientes de la las Cajas Municipales de Ahorro y Crédito del Perú.

\subsection{Calidad de servicio en el sistema financiero}

En las últimas décadas, la calidad del servicio ha recibido una gran atención tanto de académicos como profesionales, la expectativa del cliente sirve como base de la calidad del servicio. Asimismo, en el sector servicios el modelo SERVQUAL se ha utilizado con mucho éxito para evaluar su calidad. Varios investigadores han usado el modelo SERVQUAL para medir la calidad del servicio en el sector financiero (Rodríguez, 2014; Berdugo-Correa et al., 2016). De otro lado, casi todos los modelos para medir la calidad del servicio han sido criticados por presentar dificultades en su medición (De Pedro, 2015). Con el paso del tiempo, el modelo SERVQUAL fue ampliado por varios investigadores (Ibarra et al., 2014; Numpaque-Pacabaque \& RochaBuelvas, 2016; Valencia, Cruz, \& Ospino, 2018)

\subsection{Modelo de discrepancias - SERVQUAL}

Todas las organizaciones necesitan conocer la calidad del servicio que ofrecen. Asimismo, las ventajas de lograr una buena calidad de servicio son: mejorar la fidelidad del cliente e incrementar la participación del mercado (Zeithaml et al., 1996). Por lo tanto, para la medición de la calidad del servicio en las cajas municipales se ha utilizado el modelo SERVQUAL. Este modelo fue propuesto por Parasuraman et al. (1985) en sus inicios, comprendía diez dimensiones y al pasar los años se fue agrupando en cinco dimensiones. Estos constructos más importantes fueron: tangibilidad, confiabilidad, seguridad, empatía y capacidad de respuesta: a) Tangibilidad, es la apariencia o como se perciben las instalaciones físicas, el personal, los equipos y los materiales. b) Fiabilidad, es la habilidad de ejecutar el servicio prometido de manera cuidadosa y generar confianza en el cliente. c) La seguridad se refiere al conocimiento del servicio y la percepción de la atención de los trabajadores, la cortesía y su capacidad para inspirar credibilidad y seguridad en el cliente. d) Empatía, es la capacidad de atención personalizada, adaptarse al cliente, ponerse en su lugar y así mejorar su experiencia en el servicio. e) Capacidad de respuesta, es la disposición de guiar a los clientes, parecer 
totalmente comprometidos, prestar total atención a los clientes y responder las dudas o quejas durante el servicio.

El modelo SERVQUAL a pesar de ser muy utilizado en el marketing de servicio, ha recibido críticas por parte de expertos de diferentes especialidades (Fernández \& Bajac, 2018), este modelo ha tenido aplicación tanto en Estados Unidos como en Europa (De Abreu et al., 2019), además se ha empleado el modelo SERVQUAL para investigar la calidad del servicio en el contexto del sistema financiero (Fernández, 2000), Por otro lado Raajpoot (2004) propone el modelo PAKSERV donde valida tres dimensiones del modelo SERVQUAL (tangibilidad, confiabilidad y seguridad) y adiciona tres dimensiones nuevas. Ademas se ha demostrado una relación significativa del modelo SERVQUAL con la satisfacción del cliente y la lealtad del cliente (Alnaser et al., 2018), teniendo en cuenta estas contribuciones, para el presente estudio solo se consideran las tres dimensiones del modelo SERVQUAL que fueron utilizadas en el modelo PAKSERV y se proponen las siguientes hipótesis:

H1: la tangibilidad influye significativamente en la satisfacción del cliente

H2: la fiabilidad influye significativamente en la satisfacción del cliente

H3: la seguridad influye significativamente en la satisfacción del cliente

\subsection{Actitud del cliente}

La intención de comportamiento se define como la disposición del consumidor y la probabilidad de usar un servicio (San Martín \& Prodanova, 2014). Estudios previos identificaron muchos factores que podrían afectar la intención del comportamiento. Ajzen y Fishbein (1980) exponen en la teoría de la acción razonada (TRA) tanto la actitud como las normas subjetivas son capaces de afectar la intención del comportamiento humano.

Según el TRA, la actitud consiste en creencias actitudinales sobre las consecuencias de realizar el comportamiento ponderado por la valoración de las consecuencias (Aldás et al., 2011; Ajzen \& Fishbein, 1980), mientras tanto, la norma subjetiva se define como: la percepción de la persona de que la mayoría de las personas que son importantes para él o ella piensan que debería o no realizar el comportamiento en cuestión (Reyes, 2007, p. 15). De igual modo, se plantea la hipótesis.

H4: la actitud del cliente influye significativamente en la satisfacción del cliente.

\subsection{Satisfacción del cliente}

La satisfacción se atribuye a los sentimientos de felicidad del cliente cuando el proveedor del servicio satisface sus expectativas. En la literatura sobre gestión de servicios, la satisfacción del cliente puede definirse como un resumen de la reacción cognitiva y afectiva ante un incidente de servicio o una relación de servicio a largo plazo (Gosso, 2010). Según Kotler et al. (2017), la satisfacción del cliente es la respuesta del cliente que evalúa sus expectativas anteriores y el rendimiento real del servicio. Internacionalmente se ha tomado a la satisfacción del cliente como un componente muy valioso para que una organización sea considerada competitiva (Moros \& Pimiento, 2014), por otro lado, la lealtad de por vida del cliente con el servicio ofrecido depende en gran medida de su satisfacción (Kotler et al., 2017), asimismo, la calidad del servicio se ha identificado como estrategia clave para un mayor nivel de satisfacción del cliente (Guerrero, 2014), por lo cual, según Gosso (2010), menciona 
que tanto la satisfacción del cliente como la percepción de la calidad del servicio tienen un impacto positivo en la intención de recompra del cliente. En ese mismo sentido se plantea la hipótesis.

H5: la satisfacción del cliente influye significativamente en la lealtad del cliente.

\subsection{Lealtad del cliente}

La calidad del servicio es una herramienta efectiva para mantener a los clientes leales a una organización, además según Baptista y León, (2013), la lealtad es una actitud y un comportamiento específico. Asimismo, la lealtad del cliente ha sido un elemento importante para aumentar la rentabilidad de la empresa (Gosso, 2010), también, la lealtad del cliente se ha definido como un compromiso profundamente arraigado para volver a comprar o volver a patrocinar un producto preferido de manera consistente en las futuras influencias situacionales y los esfuerzos de marketing que podrían causar un cambio de comportamiento (Kotler et al., 2017, p.104).

Sin importar el tipo de medición que se realice, se ha demostrado que la calidad se relaciona positivamente con la satisfacción y lealtad de los clientes (Baptista \& León, 2013). Por otro lado, la lealtad de comportamiento refleja la respuesta positiva del cliente para repetir la comprar un producto o servicio en particular (Cavazos, 2010), es decir, los clientes que son leales a una institución financiera gastan mucho más que otros clientes (Vargas \& Aldana, 2014). Es por ello que varios estudios confirmaron que la lealtad en el sector bancario se ha atribuido en función de la satisfacción del cliente (León, 2018; Arancibia et al., 2013; Berdugo-Correa et al., 2016). Con los fundamentos mencionados se plantea el modelo teórico que se muestra en la figura 1.

\section{Figura 1. Modelo general teórico}

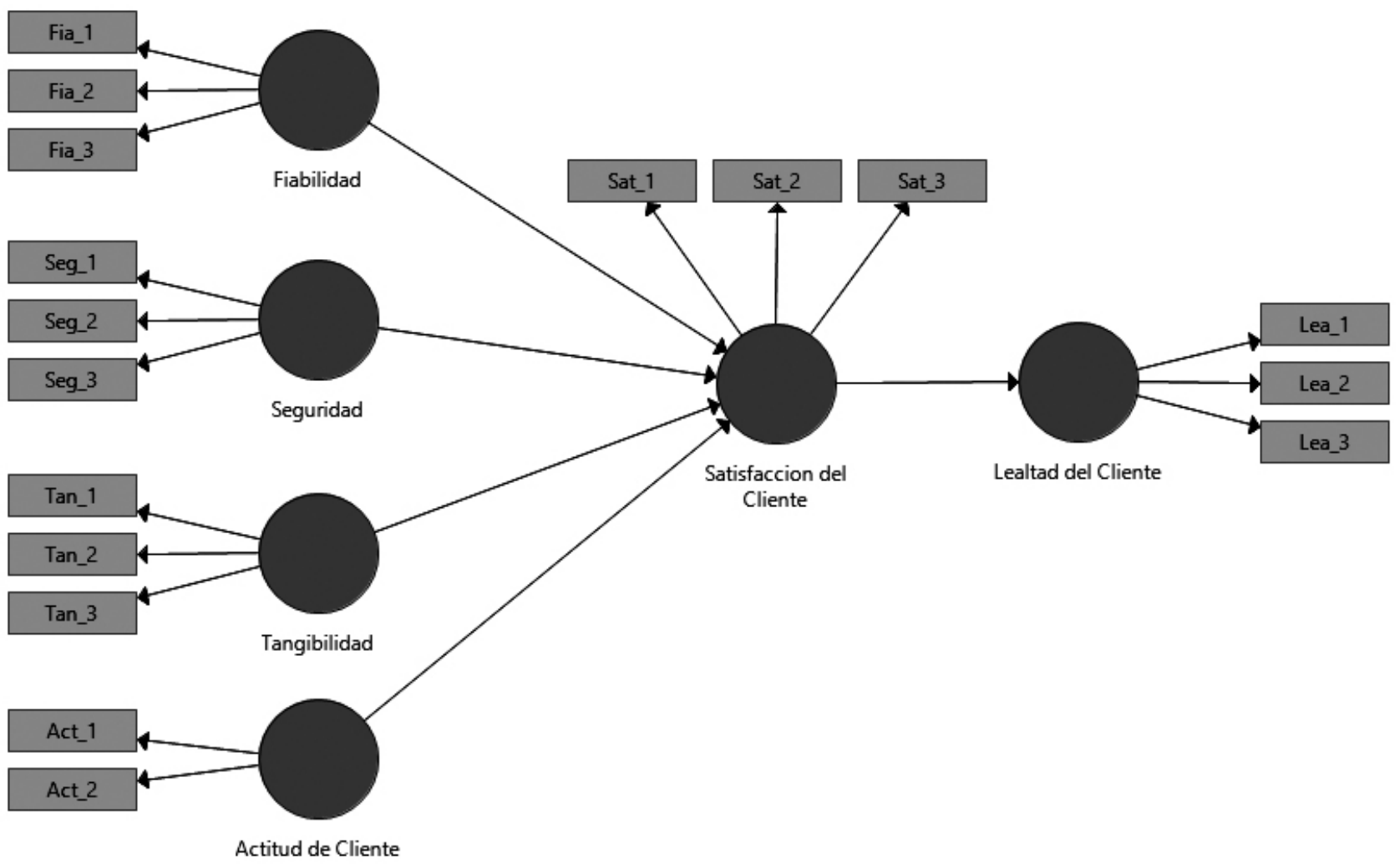




\section{Materiales y método}

El presente estudio se ha propuesto analizar la calidad del servicio en las Cajas Municipales de Ahorro y Crédito del Perú, ampliando el modelo SERVQUAL, con la adición de la actitud del cliente, considerando los aportes y críticas sobre este modelo. En la investigación se utilizó tres dimensiones del modelo SERVQUAL, propuesto en un contexto no occidental (Raajpoot, 2004). Por lo tanto, el modelo tendría seis variables latentes (fiabilidad, seguridad, tangibilidad, actitud del cliente, satisfacción del cliente y la lealtad del cliente) y diecisiete variables observables (figura 1). El tipo de investigación fue explicativo de enfoque cuantitativo, con diseño transversal (Hernández-Sampieri \& Mendoza, 2018).

\subsection{Instrumentos de recolección de datos}

Se utilizaron dos cuestionarios; uno comprendía tres constructos del modelo SERVQUAL, tenían en total nueve ítems y fueron adaptadas de la escala desarrollada previamente por (Parasuraman et al., 1988), seguido de la actitud del cliente que comprendía dos ítems adaptados del estudio anterior propuesto por Alnaser et al. (2018); a continuación, tres ítems correspondientes a la satisfacción del cliente adaptados por Sayani (2015), y finalmente, la lealtad de los clientes con tres ítems propuestos con anterioridad por Rahi et al. (2017). La segunda parte estaba compuesta por los datos demográficos de los clientes de las CMAC's como: edad, sexo y nivel de educación.

\subsection{Diseño del muestreo}

El contexto del estudio fueron las Cajas Municipales de Ahorro y Crédito, la encuesta fue autoadministrada para recopilar datos de clientes ubicados en las ciudades de Huaraz, Caraz y Chimbote de la Región Ancash del Perú. Antes de realizar la encuesta, el investigador obtuvo el permiso del gerente de cada agencia para recopilar los datos dentro de las cajas municipales. Se ha utilizado en este estudio el método del muestreo aleatorio simple. Según Hernández-Sampieri y Mendoza (2018) el muestreo aleatorio simple garantiza que la muestra sea representativa por que mantiene la aleatoriedad y se toma de manera equitativa, por lo tanto, para la recopilación de datos, los encuestadores visitaron personalmente las agencias de las cajas municipales y solicitaron a los clientes que llenaran el cuestionario, el tamaño de muestra requerido fue de 391 clientes, el proceso de recopilación de datos se ejecutó durante el mes de noviembre del 2019, incluyendo las provincias de Caraz, Yungay, Carhuaz, Huaraz y Chimbote de la Región Ancash.

\subsection{Perfil del encuestado}

Los resultados demográficos de la muestra seleccionada fueron, varones $56.25 \%$ y mujeres $43.75 \%$. La edad de los encuestados: 7.0\% son menores de 25 años, $23.4 \%$ se encuentra en el rango de 26 a 35 años, $42.2 \%$ entre 36 a 45 años y $27.4 \%$ son mayores de 46 años. Además, el nivel de educación de los encuestados, el 19\% de los encuestados tenía educación secundaria incompleta, el 48\% tenían educación secundaria completa, el 26\% de los encuestados tenían un título universitario, el 6\% de educación de nivel de maestría. Con respecto al tipo de actividad el $52 \%$ se dedica al sector servicio, $24 \%$ a la manufactura, 13\% se dedican a actividades afines a la agricultura y el 11\% son trabajadores dependientes (sector público y privado). 


\section{Resultados}

\subsection{Modelo de medición}

Para evaluar el modelo de investigación, se empleó la técnica de análisis de Mínimo Cuadrado Parcial (PLS) mediante el uso del software Smart PLS 3.3.0 (Sarstedt \& Cheah, 2019), la figura 2 muestra los resultados de las cargas factoriales según lo recomendado por el nivel umbral de Henseler et al. (2009) de 0.6. Todos los valores estaban por encima de 0.6 , lo que muestra la validez convergente del modelo.

\section{Figura 2. Modelo de Investigación}

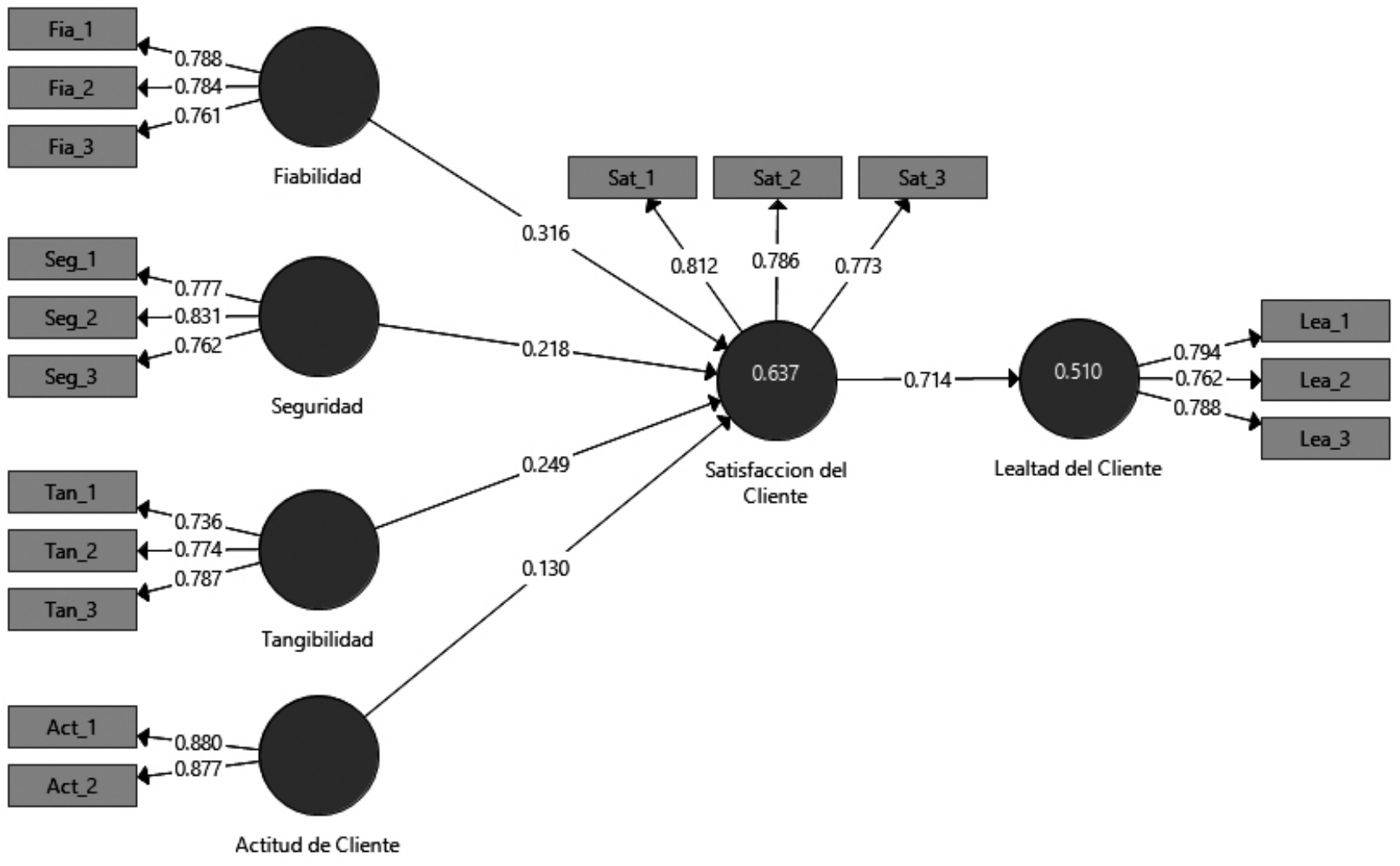

\subsection{Validez convergente}

Para evaluar la validez convergente se debe tener en cuenta la varianza promedio extraída (AVE), lo cual debería ser un valor más alto que 0.5 (Becker et al., 2018). Adicionalmente en la tabla 1 se presenta el grado de confiabilidad compuesta (CR) donde el indicador (variables observables) del constructo representa a la variable latente (Esposito et al., 2010) los valores superaron el 0.70 y se demuestra la validez convergente. 
Tabla 1. Resultados del modelo de medición

\begin{tabular}{|l|l|c|c|}
\hline \multicolumn{1}{|c|}{ Constructo } & \multicolumn{1}{c|}{$\begin{array}{c}\text { Cargas } \\
\text { externas }\end{array}$} & $\begin{array}{c}\text { Fiabilidad } \\
\text { compuesta (CR) }\end{array}$ & $\begin{array}{c}\text { Varianza media } \\
\text { extraída (AVE) }\end{array}$ \\
\hline Actitud del cliente & Act & 0.871 & 0.772 \\
\hline Act_1 & 0.880 & & \\
\hline Act_2 & 0.877 & 0.821 & \\
\hline Fiabilidad & Fia & & \\
\hline Fia_1 & 0.788 & & \\
\hline Fia_2 & 0.784 & & \\
\hline Fia_3 & 0.761 & & \\
\hline Seguridad & Gar & & \\
\hline Seg_1 & 0.777 & & \\
\hline Seg_2 & 0.831 & & \\
\hline Seg_3 & 0.762 & & \\
\hline Tangibilidad & Tan & & \\
\hline Tan_1 & 0.736 & & \\
\hline Tan_2 & 0.774 & & \\
\hline Tan_3 & 0.787 & & \\
\hline Satisfacción del Cliente & Sat & & \\
\hline Sat_1 & 0.812 & & \\
\hline Sat_2 & 0.786 & & \\
\hline Sat_3 & 0.773 & & \\
\hline Lealtad del Cliente & Lea & & \\
\hline Lea_1 & 0.794 & & \\
\hline Lea_2 & 0.762 & & \\
\hline Lea_3 & 0.788 & & \\
\hline & & & \\
\hline
\end{tabular}

\subsection{Validez discriminante}

La validez discriminante es el grado en que los elementos diferencian entre constructos, dicho de otro modo, indica en qué medida un constructo determinado es diferente de otros constructos (Martínez \& Fierro, 2018), la tabla 2 muestra la raíz cuadrada de la varianza media extraída en negrita y están en las diagonales, este valor fue mayor que los valores de cada fila y cada columna correspondientes. 
Tabla 2. Validez discriminante del modelo de medición

\begin{tabular}{|c|c|c|c|c|c|c|}
\hline Constructo & 导 & 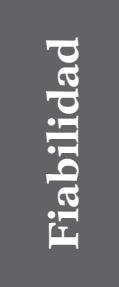 & 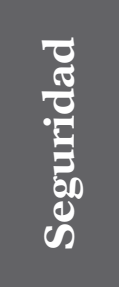 & 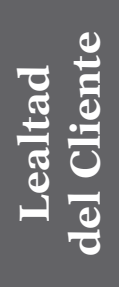 & 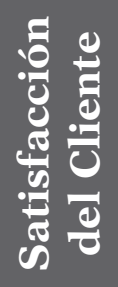 & 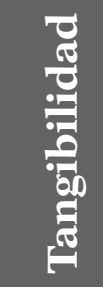 \\
\hline Actitud & 0.878 & & & & & \\
\hline Fiabilidad & 0.619 & 0.778 & & & & \\
\hline Seguridad & 0.668 & 0.699 & 0.791 & & & \\
\hline Lealtad del Cliente & 0.609 & 0.683 & 0.663 & 0.781 & & \\
\hline Satisfacción del Cliente & 0.625 & 0.722 & 0.699 & 0.714 & 0.791 & \\
\hline Tangibilidad & 0.614 & 0.693 & 0.690 & 0.662 & 0.699 & 0.766 \\
\hline
\end{tabular}

\subsection{Carga cruzada}

La validez discriminante se puede medir examinando las cargas factoriales cruzadas de las variables observadas-indicadores (Leyva \& Trinidad, 2014), se puede hacer comparando las cargas externas de un indicador en los constructos asociados y debe ser mayor que toda su carga en los otros constructos (Ruiz et al., 2010). La tabla 3 muestra que en todos los ítems que miden un constructo en particular sus cargas obtienen valores más altos en sus respectivas variables latentes y se obtienen valores más abajo en las otras variables latentes. De modo que, confirman la validez discriminante de los constructos.

Tabla 3. Carga factoriales cruzadas

\begin{tabular}{|c|c|c|c|c|c|c|}
\hline है & 을 & 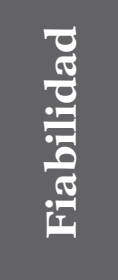 & 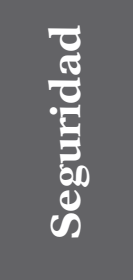 & 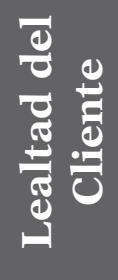 & 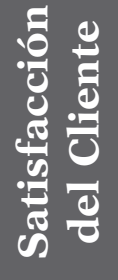 & 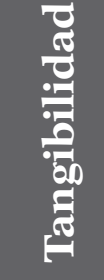 \\
\hline Act_1 & 0.880 & 0.566 & 0.597 & 0.546 & 0.552 & 0.514 \\
\hline Act_2 & 0.877 & 0.521 & 0.576 & 0.524 & 0.545 & 0.565 \\
\hline Fia_1 & 0.517 & 0.788 & 0.589 & 0.545 & 0.590 & 0.538 \\
\hline Fia_2 & 0.439 & 0.784 & 0.540 & 0.534 & 0.562 & 0.543 \\
\hline Fia_3 & 0.488 & 0.761 & 0.499 & 0.513 & 0.532 & 0.537 \\
\hline Seg_1 & 0.541 & 0.563 & 0.777 & 0.531 & 0.533 & 0.544 \\
\hline Seg_2 & 0.533 & 0.580 & 0.831 & 0.547 & 0.602 & 0.574 \\
\hline Seg_3 & 0.511 & 0.515 & 0.762 & 0.494 & 0.518 & 0.517 \\
\hline Lea_1 & 0.474 & 0.534 & 0.509 & 0.794 & 0.585 & 0.520 \\
\hline
\end{tabular}




\begin{tabular}{|c|c|c|c|c|c|c|}
\hline$\frac{\mathscr{D}}{0}$ & 矛 & 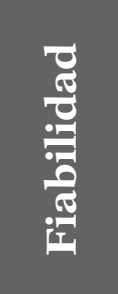 & 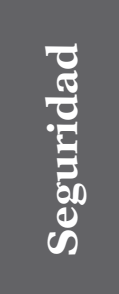 & 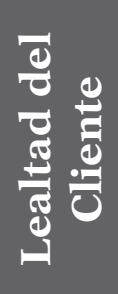 & 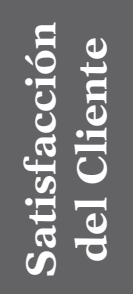 & 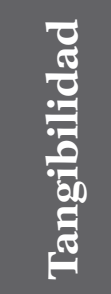 \\
\hline Lea_2 & 0.458 & 0.525 & 0.544 & 0.762 & 0.531 & 0.504 \\
\hline Lea_3 & 0.496 & 0.542 & 0.504 & 0.788 & 0.557 & 0.528 \\
\hline Sat_1 & 0.504 & 0.571 & 0.583 & 0.615 & 0.812 & 0.577 \\
\hline Sat_2 & 0.504 & 0.578 & 0.564 & 0.567 & 0.786 & 0.550 \\
\hline Sat_3 & 0.473 & 0.565 & 0.507 & 0.507 & 0.773 & 0.530 \\
\hline Tan_1 & 0.420 & 0.488 & 0.477 & 0.448 & 0.513 & 0.736 \\
\hline Tan_2 & 0.500 & 0.560 & 0.550 & 0.517 & 0.559 & 0.774 \\
\hline Tan_3 & 0.488 & 0.541 & 0.557 & 0.554 & 0.533 & 0.787 \\
\hline
\end{tabular}

\subsection{Modelo de ecuaciones estructurales (SEM)}

Después de lograr el modelo de medición, las hipótesis se probaron ejecutando un proceso de remuestreo o bootstraping mediante un numero de submuestras de 1000, como lo sugirieren Hair et al. (2017). La tabla 4 muestra los resultados de la contrastación de hipótesis, se puede ver que las cinco hipótesis tienen una influencia significativa con sus respectivas variables latentes. La tangibilidad influye significativamente en la satisfacción del cliente, $\mathrm{H1}$ : $(\beta=0.249, \mathrm{t}=4.976, \mathrm{p}<0.001)$; del mismo modo, la fiabilidad influye significativamente en la satisfacción del cliente, $\mathrm{H} 2$ : $(\beta=0.316, \mathrm{t}=5.676, \mathrm{p}$ $<0.001$ ); La hipótesis $\mathrm{H} 3$, la seguridad influye significativamente en la satisfacción del cliente, está respaldada por $(\beta=0.218, \mathrm{t}=4.553, \mathrm{p}<0.001)$; De manera similar, la actitud del cliente influye significativamente en la satisfacción del cliente, $H 4:(\beta=0.130, t=$ 3.324, $\mathrm{p}<0.01$ ); para finalizar, la hipótesis $\mathrm{H} 5$ la satisfacción del cliente influye significativamente en la lealtad del cliente debido a los valores $(\beta=0.714, t=16.154, p<0.001)$. Esto demuestra la validez del modelo en el sector financiero. Además, se utilizó el $\mathrm{R}^{2}$ para medir el tamaño del efecto. El $\mathrm{R}^{2}$ para la satisfacción del cliente fue de $0.637 \mathrm{y}$ para la lealtad del cliente de 0.510 , que es aceptable según el límite sugerido por Cohen, (1988), Por último, el residuo estandarizado cuadrático medio (SRMR) fue 0,06 donde un valor de SRMR $<0,08$ es aceptable y SRMR $<0,05$ es óptimo. 
Tabla 4. Análisis del modelo estructural (prueba de hipótesis)

\begin{tabular}{|l|l|l|l|l|l|}
\hline \multicolumn{1}{|c|}{ Relación } & \multicolumn{1}{|c|}{ (1) } & & \\
\hline
\end{tabular}

\section{Discusión y conclusiones}

A pesar de existir aceptación del modelo SERVQUAL para medir la calidad del servicio, también ha servido como base para realizar algunas modificaciones o adaptaciones para generar nueva formas de medir la calidad del servicio en entornos específicos; de manera que, ha sido adaptado como la escala SERVQHOS creada para medir la calidad de los servicios de atención hospitalaria; cuenta con 19 ítems, donde combina expectativas y percepciones a fin de obtener su valoración de la calidad (Numpaque-Pacabaque \& Rocha-Buelvas, 2016), por otro lado, existe una adaptación del modelo SERVQUAL a una denominado PAKSERV donde se consideran el impacto de los valores personales y las orientaciones de una cultura asiática, concentrados en las siguientes dimensiones tangibilidad, fiabilidad, garantía, sinceridad, personalización, formalidad y capacidad de respuesta, cuanta con 24 ítems (Raajpoot, 2004).

De modo que, el propósito de este estudio fue integrar al modelo SERVQUAL la dimensión de la actitud del cliente, y los resultados revelaron que la actitud influye significativamente en la satisfacción del cliente. Además, la realidad peruana es diferente a la realidad de Estados Unidos o Europa, por lo tanto, se confirma que la actitud desempeñó un papel moderado $(\beta=0.130)$ en la satisfacción del servicio en las cajas municipales del Perú. Por otro lado, la influencia de la satisfacción del cliente en la lealtad del cliente ha resultado ser fuerte $(\beta=0.714)$. Así mismo, todas las hipótesis fueron contrastadas, estos resultados también han sido respaldados por varios investigadores (Rahi et al., 2017; Leyva \& Trinidad, 2014; Fernández, 2000),

Se han realizado varios estudios para investigar los problemas de calidad del servicio en diferentes contextos (Yalley \& Agyapong, 2017; Valencia et al., 2018). De este modo, la investigación actual tiene dos contribuciones principales. En primer lugar, los resultados de la investigación demostraron que las dimensiones del modelo SERVQUAL, 
como seguridad, tangibilidad y confiabilidad, son relevantes para la medición de la calidad del servicio en las cajas municipales del Perú. En segundo lugar, este estudio también amplió las dimensiones del modelo SERVQUAL con la actitud del cliente para medir la calidad de servicio en el sector financiero. El modelo propuesto en la presente investigación tiene un efecto significativo en la satisfacción del cliente con un 63.7\% de variación y la satisfacción del cliente explica el 51\% de variación de la lealtad del cliente de las Cajas Municipales de Ahorro y Crédito del Perú. De manera que, los resultados del estudio confirman un nuevo modelo que expresa la cultura peruana con la añadidura de la actitud del cliente como componente de la calidad de servicio.

El creciente número de instituciones financieras como Edpymes, cajas rurales, cooperativas entre otras microfinancieras en el Perú (Escobedo et al., 2019), conforman un panorama que crea una oportunidad para que las cajas municipales se involucren de forma integral en mejorar las relaciones con sus clientes y encontrar nuevas formas de generar valor para los clientes. De acuerdo con los resultados del modelo propuesto, la actitud cumple un papel importante en la percepción de la calidad de servicio, por lo tanto, los puntos de contacto son críticos y el funcionario de créditos debe estar bien preparado, puesto que estas interacciones pueden mejorar la relación con los clientes. No se trata únicamente de entregar información, sino de reforzar las relaciones con los clientes (Baptista \& León, 2013) y conducir a las cajas municipales a un modelo centrado en el cliente, lograrlo no será rápido ni sencillo, pero es el momento de iniciar este proceso.

Teniendo en cuenta la actitud del cliente, se sugiere que las cajas municipales deben implementar módulos de protección al consumidor y mejorar el acceso a la información de sus productos para evitar confusiones y aclarar las condiciones al momento de contratarlos, y así mejorar la percepción del cliente respecto a la calidad de servicio y generar las condiciones necesarias para que el sistema financiero continúe fuerte en beneficio de la inclusión financiera.

La principal limitación del estudio podría ser el tipo de muestra, es decir se consideró a los clientes de una región, acompañado del carácter no longitudinal del análisis. No obstante, se sugiere realizar investigaciones futuras ampliando el estudio a otras instituciones financieras o la muestra podría representar una gran diversidad de países para investigar las posibles diferencias en el comportamiento de los clientes.

\section{Referencias}

Ajzen, I., \& Fishbein, M. (1980). Understanding attitudes and predicting social behavior. Prentice-Hall, Inc. Aldás, J., Lassala, C., Ruiz, C., \& Sanz, S. (2011). Análisis de los factores determinantes de la lealtad hacia los servicios bancarios online. Cuadernos de Economía y Dirección de la Empresa, 14(1), 26-39. https://doi.org/10.1016/j.cede.2011.01.003

Alnaser, M. I., Ghani, M., \& Rahi, S. (2018). Service qualityin Islamic banks: The role of PAKSERV model, customer satisfaction and customer loyalty. Accounting, 4(2), 63-72. https://doi.org/10.5267/j.ac.2017.8.001

Arancibia, S., Leguina, A., \& Espinosa, P. (2013). Factores determinantes en la percepción de la imagen y calidad de servicio y sus efectos en la satisfacción del cliente: Un caso aplicado a la banca chilena. Revista de Ciencias Social, 19(2), 255-267.

Baptista, M.V., \& León, M.d. (2013). Estrategias de lealtad de clientes en la banca universal. Estudios Gerenciales, 29(127), 189-203. https://doi.org/10.1016/j.estger.2013.05.007

Becker, J.M., Ringle, C., \& Sarstedt, M. (2018). Estimating moderating effects in PLS-SEM and 
PLSc-SEM: Interaction term generation* data treatment. Journal of Applied Structural Equation Modeling, 2(2), 1-21.

Berdugo-Correa, C., Barbosa-Correa, R., \& Prada-Angarita, L. (2016). Variables relevantes para la medición de la calidad percibida del servicio bancario. Dyna, 83(197), 212-221. http://dx.doi.org/10.15446/dyna.v83n197.55426

Cavazos, J. (2010). El Concepto de Marketing Bajo el Paradigma Relacional. Una Agenda para Latinoamérica. Revista Brasileira de Marketing, 8(1), 5-23. https://doi.org/10.5585/remark.v8i1.2124

Cohen, J. (1988). Statistical power analysis for the social sciences. Lawrence Earlbaum Associates.

De Abreu, A.A., Antonialli, L.M., \& Andrade, D.M. (2019). Xplorando a base intelectual do tema qualidade em serviços: quando o discurso muda o tom. Brazilian Journal of Marketing, 18(2), 137 168. https://doi.org/10.5585/remark.v18i2.3968

De Pedro, P.E. (2015). La calidad de servicio bancario: una escala de medición. (Tesis de Maestría). Universidad Nacional del Sur, Bahía Blanca, Argentina.

Escobedo, H.J., Luque, V.J., Ríos, W. I., \& Tapia, M. (2019). Medición de la calidad en el servicio de la Caja Municipal Cusco en la ciudad de Cusco. (Tesis de maestría). Pontificia Universidad Católica del Perú, Lima.

Esposito, V., Chin, W.W., Henseler, J., \& Wang, H. (2010). Handbook of partial least squares: Concepts, methods and applications. Springer.

Fernández, M. (2000). Validación de SERVQUAL como instrumento de medida de la calidad del servicio bancario. Revista Europea de Dirección y Economía de la Empresa, 9(1), 57-70.

Fernández, P., \& Bajac, H. (2018). Gestión del marketing de servicios: Principios y aplicaciones para la actividad gerencial. Ediciones Granica S.A.

Geraldo, L.A., Soria, J.J., \& Tito, P.L. (2020). Modelo SEM basado en valores organizacionales y capital intelectual: un estudio realizado en entidades del sistema financiero peruano. Retos, Revista de Ciencias de la Administración y Economía, 10(19), 5-28. https://doi.org/10.17163/ret.n19.2020.01

González, R. (2015). Evaluación de la calidad del servicio percibida en entidades bancarias a través de la escala Servqual. Ciencia e Ingeniería Neogranadina, 25(1), 113-135. https://doi.org/10.18359/rcin.439

Gosso, F. (2010). Hiper satisfacción del cliente: Conceptos y herramientas para ofrecer un servicio sobresaliente. Panorama editorial.

Guerrero, G.R. (2014). La calidad del servicio al cliente en los grandes supermercados de Ibagué: un análisis desde la escala multidimensional (SERVQUAL). Cuadernos de Administración, 30(52), 54-64. https://bit.ly/2Sm7akg

Hair, J., Hult, T., Ringle, C., \& Sarstedt, M. (2017). A Primer on Partial Least Square Structural Equation Modeling (PLS-SEM) (2da ed.). Sage Publications, inc.

Henseler, J., Ringle, C., \& Sinkovics, R. (2009). The use of partial least squares path modeling in international marketing. En R. Sinkovics, y P. Ghauri, New Challenges to International Marketing. Advances in International Marketing, 20, 277-319. https://doi.org/10.1108/S1474-7979(2009)0000020014

Hernández-Sampieri, R., \& Mendoza, C. (2018). Metodología de la investigación. Las rutas cuantitativa, cualitativa y mixta. Mc Graw Hill.

Ibarra, L.E., Espinoza, B., \& Casas, V. (2014). Aplicación del modelo Servqual para evaluar la calidad en el servicio en los hospitales públicos de Hermosillo, Sonora. Tecnociencia Chihuahua, 8(2), 98-108. https://bit.ly/31ko0Uh

Kotler, P., Bowen, J.T., Makens, J., \& Baloglu, S. (2017). Marketing for hospitality and tourism. (7ma ed.). Pearson Education.

León, J. (2018). Inclusión financiera de las microempresas y las pequeñas y medianas empresas en el Perú: el caso de la banca de desarrollo. En E. Pérez, y D. Titelman, La inclusión financiera para la inserción productiva y el papel de la banca de desarrollo (pp. 189-219). CEPAL.

Leyva, O., \& Trinidad, J. (2014). Modelo de ecuaciones estructurales por el método de mínimos cuadrados parciales (Partial Least Squares-PLS). En K. Sáenz, y G. Tamez, Métodos y técnicas 
cualitativas y cuantitativas aplicables a la investigación en ciencias sociales (pp. 480-497). Tirant Humanidades. https://bit.ly/2VR6x4r

Martínez, M., \& Fierro, E. (2018). Aplicación de la técnica PLS-SEM en la gestión del conocimiento: un enfoque técnico práctico. RIDE. Revista Iberoamericana para la Investigación y el Desarrollo Educativo, 8(16), 130-164. https://doi.org/10.23913/ride.v8i16.336

Morillo, M. d., Morillo, M.C., \& Rivas, D.E. (2011). Medición de la calidad del servicio en las instituciones financieras a través de la escala de Servqual. Contaduría y administración (234), 101-130.

Moros, M.A., \& Pimiento, E.O. (2014). Impacto de las TIC en la calidad de servicio y satisfacción de los clientes como herramienta de competitividad en el sector financiero. Caso de estudio: banco sofitasa-venezuela. En M.V. Flores, A.V. López, y E.A. Chávez, La productividad, competitividad y capital humano en las organizaciones (pp. 439-468). Ediciones Ilsa S.A.

Numpaque-Pacabaque, A., \& Rocha-Buelvas, A. (2016). Modelos SERVQUAL y SERVQHOS para la evaluación de calidad de los servicios de salud. Revista de la Facultad de Medicina, 64(4), 715720. http://dx.doi.org/10.15446/revfacmed.v64n4.54839

Parasuraman, A., Zeithaml, V.A., \& Berry, L. L. (1985). A conceptual model of service quality and its implications for future research. Journal of marketing, 49(4), 41-50.

Parasuraman, A., Zeithaml, V. A., \& Berry, L. L. (1988). Servqual: A multiple-item scale for measuring consumer perceptions of service quality. Journal of retailing, 64(1), 12. https://bit.ly/31KQ84d

Raajpoot, N. (2004). Reconceptualizing service encounter quality in a non-western context. Journal of Service Research, 7(2), 181-201. https://doi.org/10.1177/1094670504268450

Rahi, S., Yasin, N., \& Alnaser, F. (2017). Measuring the role of website design, assurance, customer service and brand image towards customer loyalty and intention to adopt internet banking. The Journal of Internet Banking and Commerce, S8(024). https://bit.ly/2VO1oKi

Reyes, L. (2007). La Teoría de la Acción Razonada. Implicaciones para el estudio de las actitudes. Investigación educativa duranguense, (7), 66-77. https://bit.ly/2YQ89fL

Rodríguez, V. (2014). Dinero electrónico en Perú: ¿Por qué es importante en la inclusión financiera? Quipukamayoc, 22(41), 175-192. https://doi.org/10.15381/quipu.v22i41.10084

Ruiz, M.A., Pardo, A., \& San Martín, R. (2010). Modelos de ecuaciones estructurales. Papeles del psicólogo, 31(1), 34-45. https://bit.ly/2ye0Ap2

Salinas, J. (2011). Ecuador, microcrédito: ¿Negocio o inclusión financiera? Retos, Revista de Ciencias de la Administración y Economía, 1(2), 125-134. https://doi.org/10.17163/ret.n2.2011.06

San Martín, S., \& Prodanova, J. (2014). ¿Qué factores fomentan la compra por impulso en el comercio móvil? Revista Española de Investigación de Marketing ESIC, 18(1), 32-42. https://doi.org/10.1016/s1138-1442(14)60004-8

Sarstedt, M., \& Cheah, J.H. (2019). Partial least squares structural equation modeling using SmartPLS: a software review. Journal of Marketing Analytics, 7(3), 162-202. https://doi.org/10.1057/s41270-019-00058-3

Sayani, H. (2015). Customer satisfaction and loyalty in the United Arab Emirates banking industry. International Journal of Bank Marketing, 33(3), 351-375. https://doi.org/10.1108/IJBM-12-2013-0148

Ureña, Y.C., Quiñones, E., \& Carruyo, N. (2016). Capital intelectual: modelo estratégico para la calidad de servicio en organizaciones inteligentes. Revista Orbis (35), 3-17. https://bit.ly/2yhxQvs

Valencia, V.E., Cruz, S., \& Ospino, Ó.J. (2018). Aplicación del modelo SERVQUAL para la determinación de los factores que inciden en la satisfacción de los usuarios en los hogares infantiles de Chibolo, Magdalena. INGE CUC, 14(2), 19-27. https://doi.org/10.17981/ingecuc.14.2.2018.02

Vargas, M.E., \& Aldana, L. (2014). Calidad y servicio: conceptos y herramientas (3ra ed.). Ecoe Ediciones.

Yalley, A.A., \& Agyapong, G.K. (2017). Measuring service quality in Ghana: a crossvergence cultural perspective. Journal of Financial Services Marketing, 22(2), 43-53. https://doi.org/10.1057/s41264-017-0021-x

Zeithaml, V.A., Berry, L.L., \& Parasuraman, A. (1996). The behavioral consequences of service quality. Journal of marketing, 60(2), 31-46. https://doi.org/10.2307/1251929 